\title{
Face Identification based on Contourlet Transform and Multi-layer Perceptron Classifier
}

\author{
Ali Fattah Hassoon \\ Electrical Engineering \\ Department \\ College of Engineering \\ Mustansiria University \\ Baghdad-Iraq
}

\author{
Maher K. AL-Azzawi \\ Electrical Engineering \\ Department \\ College of Engineering \\ Mustansiria University \\ Baghdad-Iraq
}

\author{
Tariq Tashan, $\mathrm{PhD}$ \\ Electrical Engineering \\ Department \\ College of Engineering \\ Mustansiria University \\ Baghdad-Iraq
}

\begin{abstract}
Useful properties of the Contourlet Transform (CT) are exploited in this paper to investigate more discriminant features to enhance the face identification performance. In this paper a face identification system is suggested based on CT, and Multi-Layer Perceptron (MLP) Classifier. The main reasons behind using the $\mathrm{CT}$ are: First, the $\mathrm{CT}$ supports progressive data compression/expansion, hence it is used for image compression. Second, since the features in human face are not just horizontal or vertical. CT is utilized for feature extraction because it is a genuine 2-D transform that can capture the edge information in all directions. After decomposing an image by CT, low and high frequency coefficients of CT are calculated in different scales and various angles. The frequency coefficients are utilized as an input feature vector for a neural network classifier. Simple feed forward MLP neural network is used to achieve the identification process. The network parameters are tuned to optimal values, in order to produce fair comparison between different types of feature vectors. To evaluate the algorithm performance five different databases are used. Some of them of high variability, which examines the algorithm robustness against variability. In addition, the proposed algorithm is evaluated using a generated database which composes two databases. Then the suggested method is compared to other classical feature-based methods such as, wavelet, and Principle Component Analysis (PCA). The results indicate that the CT-based method has better identification rate, and is faster than the Wavelet-based and the PCA-based methods. This is due to the high sparsity of the CT and its efficient capability of compression. An average identification rate of 93.94\% is obtained for the CT-based method, $85.12 \%$ for the Wavelet and $79.96 \%$ for the PCA.
\end{abstract}

\section{Keywords}

Contourlet Transform, Face Identification, Multi-Layer Perceptron Neural Network.

\section{INTRODUCTION}

Face Recognition is classified into two types, face identification and face verification. Face identification is a close-set problem (one-to-many matching), where the objective is to identify a person using his/her face image, from a specific number of images. While, face verification is an open-set problem (one-to-one matching), where the objective is to verify a test image whether it belongs to the claimed person or not. Face verification is comparatively easy and realized in face code, mobile, computer login verification. Whilst, face identification is more complicated, because a large database needs to be compared [1]. Although a human can recognise faces easily, present face recognition systems are still constrained, particularly if they have to deal with the variations in expression, background, pose, age, and illumination. These obstacles make the face recognition a difficult task. Another challenging is the limited number of training samples [2].

The topic of identification has attracted engineers and specialist's minds for a long time, to design smart systems that can help to distinguish the personality of persons [3]. Two types of features can be utilized for identification process, which are: 1. Indirect features, for example, sorts of passwords fundamentally depend on account of the likelihood of simple access to individuals. These are poor and have low credits. 2. Direct features, are the features that rely on physical, mental and passionate of individuals, for example, fingerprint, signature, and handwriting. Face images are employed in face recognition problem as follows:

For face identification, a face image enters the system as an input and the system is asked to determine who is the face image belongs to. In fact, the input face image belongs to one of the existing face classes.

For face verification, the input face image is claimed to be belong to a certain person, the system, compares the input image against a reference face image(s) of the claimed person. The system decide to approve or deny the input face image.

The Eigenfaces method based on the PCA method is introduced by Turk and Pentland [4], it is one of the popular approaches widely used in face recognition. Its main idea is to find a group of orthogonal basis images (known as Eigenfaces) and due to this basis, the image coordinates (the PCA coefficients) becomes uncorrelated [2]. Moreover, there are other appearance-based approaches like Linear Discriminant Analysis (LDA) [5] and Independent Component Analysis (ICA) [6]. Both approaches yield constrained results for the face images acquired in an open air environment, with changes in illumination and/or pose. N.G.Chitaliya and A.I.Trivedi use Wavelet-PCA based feature extraction for Face Recognition system [7]. It is known that representing point singularities can be performed efficiently by using wavelets. The typical orthogonal wavelet transform provides wavelets with three orientations: vertical, horizontal and diagonal. However, for higher dimensions wavelets disregard the geometric features of objects with edges, and as a result the regularity of the edges curves is not exploited [8]. Multi-scale Geometric Analysis (MGA) is a theory has been developed for high dimensional signals. Several MGA tools are suggested like Curvelet $[9,10]$, Bandlet and Contourlet [11-15]. These features represent information of images with more directional information and smooth contour which can be done effectively by computing the CT instead of the Wavelet transform, due to its properties of directionality and 
anisotropy [2]. Ch.Srinivasa Rao uses CT as feature vector for Content Based Image Retrieval (CBIR) System [16]. Yan et al. propose a face recognition approach based on CT [17].

In this paper, a face identification method based on CT and feed-forward MLP with back propagation artificial neural network is presented. The discrete CT is used for image compression and feature extraction for the face identification systems. The reasons for using the CT are:

1. The CT provides efficient compression for full set coefficients and scaled-down version of the image.

2. In face identification, the edge data is essential in determining elements, and the edges inside of a human face are not merely horizontal or vertical.

The coefficients set of the CT are utilized as a feature vector, this feature vector is utilized as an input to the neural network classifier.

\section{FACE IDENTIFICATION SYSTEM}

In any identification system it is necessary to create appropriate database prior of processing. The first phase in identification system contains low-level operations, including preprocessing operations, such as image enhancement or noise removal. The second phase, features of prepared images and pre-selection process are extracted. The third phase includes high-level operations and comprehension of image by help of the outcomes and the extracted features. This can be done using a classifier. Methods such as fuzzy classifier, statistical, neural network can be used [3]. Fig (1) shows the structure of any face identification system.

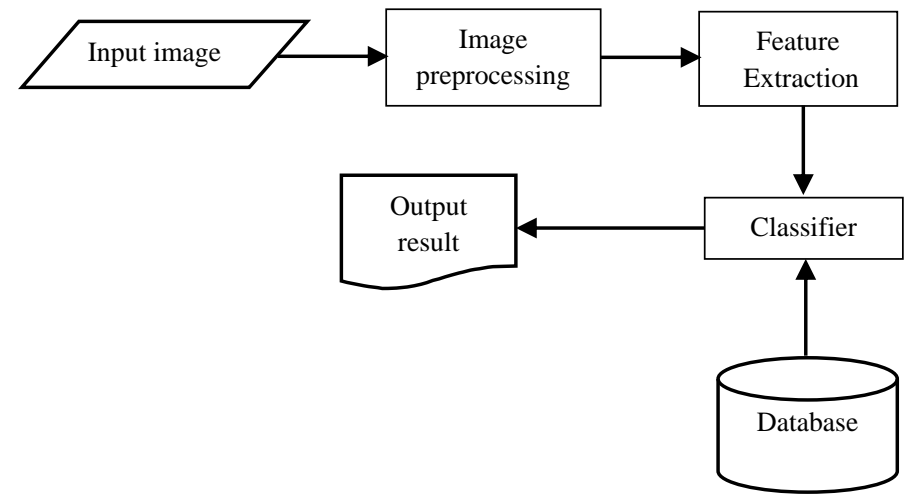

Fig 1: The structure of face identification system.

\section{CONTOURLET TRANSFORM (CT)}

Wavelet Multi-scale decomposition has an advantage on the Fourier Transform, where it captures both frequency and location information. However to represent the image that has smooth contours in different directions, wavelets are not effective, because of its limited ability of capturing directional information. In other words, 2-D multi-resolution wavelets fail when dealing with image of cartoon class, i.e. images consisting of domains of grey values vary smoothly and separated by smooth boundaries [18]. Do and Vetterli visualise the CT [19], which addresses this problem, because the CT is based on an efficient 2-D multi-scale and multidirectional filter banks, that can effectively deal with images of smooth contours. In addition, the CT provides two additional properties, directionality and anisotropy. The CT can be considered as an extension of the wavelets with directionality [18], and it is one of many of transforms developed lately, aiming to improve the representation of sparsity of images over the Wavelet Transform. Fig (2) shows the CT structure.

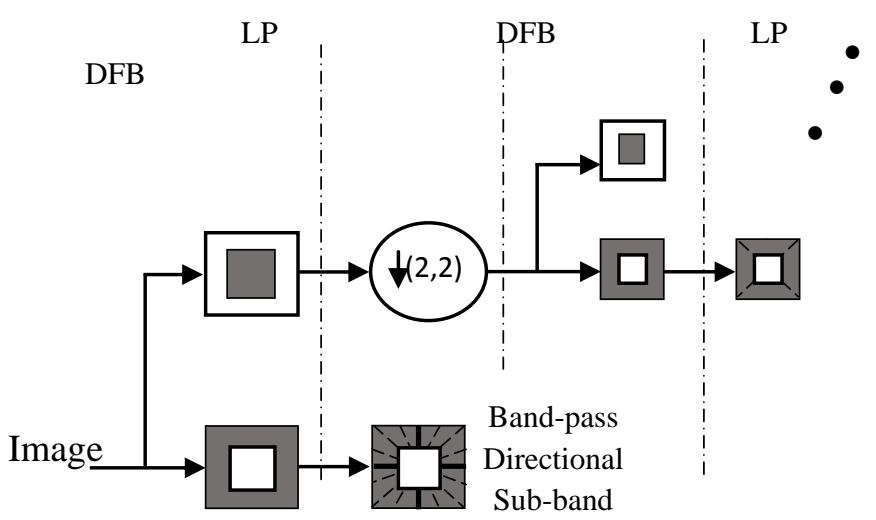

Fig 2: The CT filter structure

It is constructed from two stages. The first stage is a Laplacian Pyramid (LP) containing L+1 scale levels, and iteratively decomposes a 2-D image into low-pass and high-pass subbands. The second stage is Directional Filter Bank (DFB), which further decomposes the frequency spectrum of the high-pass sub-band of the LP. This two stage decomposition is constructed as follows: first, the points of discontinuity can be captured by using the LP, and followed by a DFB which links the points of discontinuity into linear structures. The result is an image expansion using basic elements such as contour segments, and therefore is named Contourlet. CT has ability to approximate a smooth contour at multiple resolutions in efficient way because in particular, Contourlets have extended supports at various scales, different directions, and aspect ratios. In the frequency domain, the CT provides a multi-scale and directional decomposition [2].

The CT for each level of the LP of the $j^{\text {th }}$ decomposition can produce $2^{l_{j}}$ high frequency images, where $l_{j}$ represents the number of sub-bands of the DFB. Finally, $\sum_{j=1}^{L} 2^{l_{j}}$ contourlet images are obtained, where $L$ represents the scales number of LP. For example if $l_{1}=4, l_{2}=3$ and $l_{3}=2$, this produces 16 (high frequency images on the finer level), 8 (high frequency images on the middle level), and 4 (high frequency images on the coarser level) respectively. The coefficients of the CT are coloured toward white if these coefficients are large in absolute value, while coefficients of small absolute value are coloured toward black.

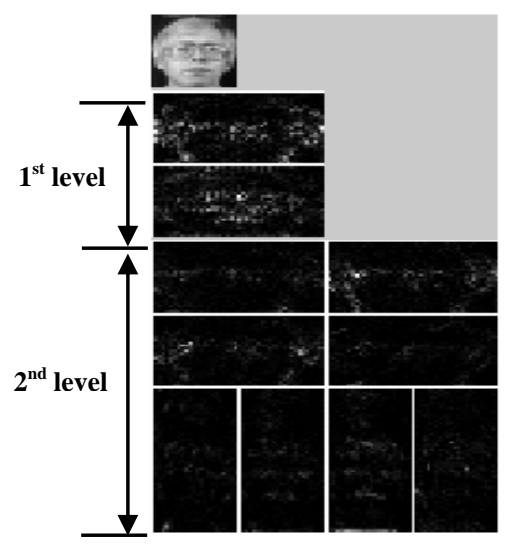

Fig 3: CT coefficients of two levels $\left(l_{1}=1, l_{2}=2\right)$ of face image 


\subsection{Laplacian Pyramid (LP)}

LP is one way of multi-scale decomposition introduced by Burt and Adelson [18]. The outputs at each level of the LP represent a down sampled low-pass version of the original image and band-pass image, which results from the difference between the original and the predicted image [18]. Fig (4) illustrates the decomposition process, where $\mathrm{H}$ is called lowpass filter in analysis stage, $G$ is called low-pass filter in synthesis stage, and $\mathrm{M}$ refers to the sampling matrix. The process can be repeated on the coarse image which is down sampled low-pass version of the original image (Low frequencies). In multi-dimensional filter banks, sampling is represented by matrices known as sampling matrices; for instance, down sampling $x[n]$ by $M$ gives $x_{d}[n]=x[M n]$, where $\mathrm{M}$ is an integer matrix.

a

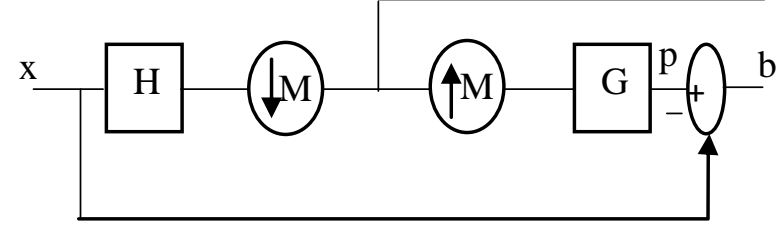

Fig 4: LP decomposition

LP shown in Fig (4) is a one-level decomposition where the outputs are coarse approximation $c[n]$ and the difference $b[n]$ between the original and predicted signal $\mathrm{p}[\mathrm{n}]$.

\subsection{Directional Filter Bank (DFB)}

The high frequency content like smooth contours and directional edges can be captured by using DFB [20]. The DFB is implemented by utilizing a $k$-level binary tree decomposition that results $2^{k}$ directional sub-bands with wedge shaped frequency partitions as illustrated in Fig (5). A simplified DFB is used in this work, which is constructed from two building blocks. The first one divides a 2-D spectrum into two directions (horizontal and vertical) this is done by using a two-channel quincunx filter bank with fan filters. The second amounts to reorder the pixels of the image by using a shearing operator. Because of these two operations, directional information is preserved. This is the desirable characteristic in CBIR system used to improve retrieval efficiency.

The combination of an LP and DFB produces a double filter bank structure called Pyramidal Directional Filter Banks (PDFB) or Contourlet filter bank. The difference images or the band-pass images result from the difference between the original images and the predicted images. These images are fed to the DFB, and as a result the directional information can be captured. The scheme can be repeated on the coarse image [20]. The PDFB decomposes the given image into directional sub-bands at multiple scales.

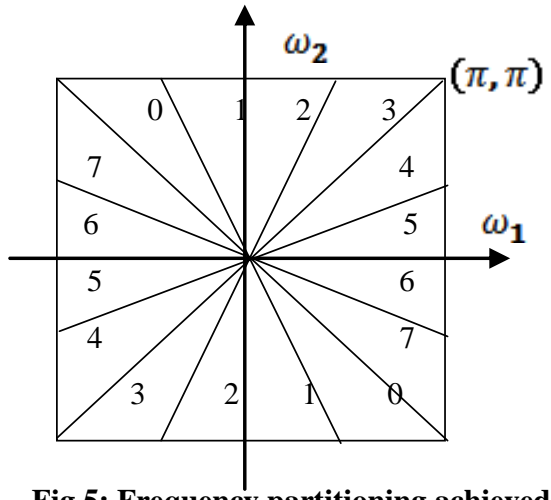

Fig 5: Frequency partitioning achieved by DFB for $k=3$ and lead to $2^{3}=8$ real wedge-shape frequency bands.

\section{CLASSIFIER}

In this paper a simple MLP neural network is suggested to apply the identification task. Fig (6) presents the suggested supervised neural network. The input layer of the neural network has the same size of the feature vector $(\mathrm{N})$, while the output layer size equals to $(\mathrm{S})$ the number of persons to be identified in the database. The size of the hidden layer is to be experimentally optimised, depending on the complexity of the identification problem. In back propagation, the learning process requires pairs of input vector $\left(\mathrm{X}_{1} \ldots \ldots \mathrm{X}_{\mathrm{N}}\right)$ and target vector $\left(\mathrm{T}_{1} \ldots \mathrm{T}_{\mathrm{S}}\right)$. The target vector $\mathrm{T}$ is compared to the actual output vector $\mathrm{Y}$. When the target vector and the actual output vector are different, the weights are adjusted to minimise the difference. Initially, the weights are assigned to random values. At each epoch, the weights are updated in order to minimise the mean square error between the actual output vector and the target vector [18]

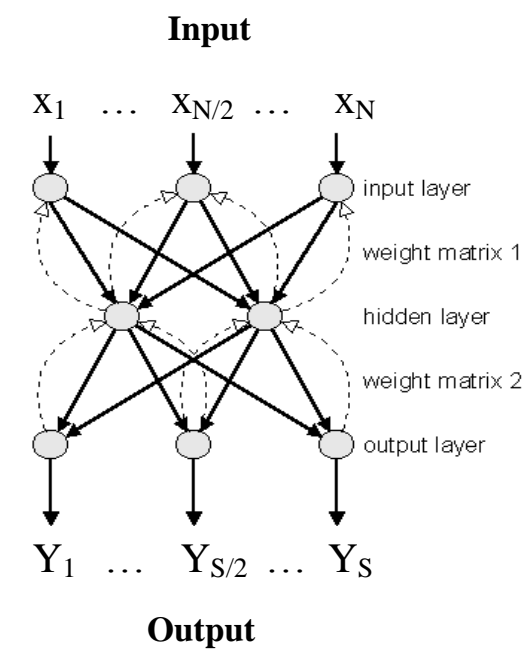

Fig 6: Structure of neural classifier.

The $m^{\text {th }}$ input of the hidden layer is given by:

$$
n e t_{m}=\sum_{i=1}^{N} X_{i} W_{(i, m)}
$$

Where $X_{i}$ is the $i^{\text {th }}$ component of the input feature vector, and $W_{(i, m)}$ is the weight that links the $i^{\text {th }}$ input node to the $m^{\text {th }}$ hidden node. The output of the $m^{\text {th }}$ hidden node is fed to a sigmoid activation function that is mostly used, and expressed as follows: 


$$
h_{m}=\frac{1}{1+\exp \left(-n e t_{m}\right)}
$$

In the same manner, the $s^{\text {th }}$ output in the output layer is given by:

$$
n e t_{s}=\sum_{i=1}^{M} h_{i} W_{(i, s)}
$$

Where $M$ is the number of hidden nodes, $h_{i}$ is the output of the $i^{\text {th }}$ hidden node, and $W_{(i, s)}$ is the weight that links the $i^{\text {th }}$ hidden node to the $s^{\text {th }}$ output. The output of the $s^{\text {th }}$ output node is given by:

$$
h_{s}=\frac{1}{1+\exp \left(-n e t_{s}\right)}
$$

To update the weights, the error between the output vector and the target vector is calculated as follows:

$$
E=\frac{1}{2} \sum_{i=1}^{S}\left(Y_{i}-T_{i}\right)^{2}
$$

$Y_{i}$ and $T_{i}$ represent the actual output and the target output, respectively. If the error is less than a predefined limit, the training process is stopped; otherwise, the error is back propagated to update the weights.

The neural classifier consists of training phase and testing phase. To train the Neural Network, the image in the training set (seen image data) is decomposed by CT (as described in section 3) then the resultant frequency coefficient is used as the input feature vector of the Neural Network. When a new image from the testing set (unseen image data) is considered for identification, the image is also decomposed by using the CT and coefficients are calculated for the certain image. The number of nodes in the output layer is equal to the number of individuals' image, to be identified. The position of the output with the highest value determines the identity to which the input belongs. For example if there are five classes [a, b, c, d, e], five outputs are needed. During the training phase, each feature vector data in the training set is presented to the classifier along with its desired output, where the desired output for an input that belongs to class $c$ is a zero vector with one at the $c^{\text {th }}$ position $[0,0,1,0,0]^{\mathrm{T}}$.

In this work, the neural network is experimentally tuned to the following parameters: a single hidden layer with 2000 number of neurons and 200 epochs. The activation function for both the hidden and output layer are logarithmic sigmoid. The network is trained using Scaled conjugate gradient back propagation method. Fig (7) shows the identification rate at different numbers of hidden nodes and epochs.

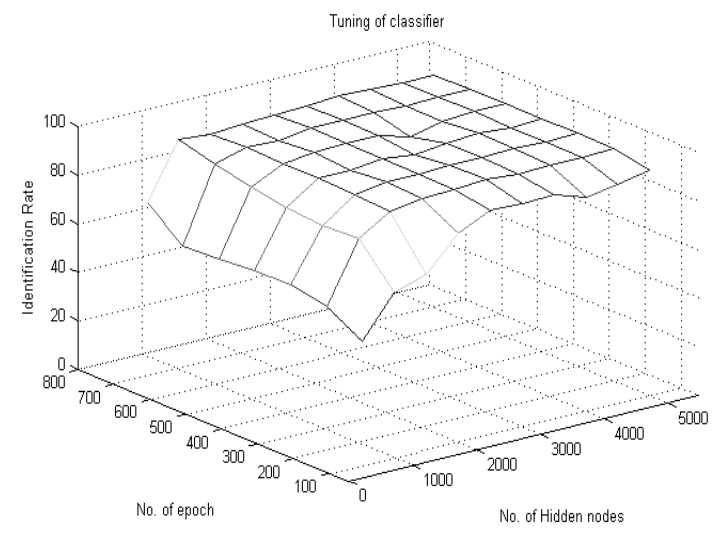

Fig 7: Tuning the parameters of classifier.

\section{DATA PREPARATION}

Five standard face recognition databases are used to evaluate the presented algorithm in this paper. These databases are summarised as follows

\subsection{Olivetti Research Laboratory (ORL)}

The AT\&T database (formally ORL) Ten different images each of 40 distinct subjects. For some subjects, the images were taken at different times, varying the lighting, facial expressions (open / closed eyes, smiling / not smiling) and facial details (glasses / no glasses). All the images were taken against a dark homogeneous background with the subjects in an upright, frontal position (with tolerance for some side movement) [8]. Fig (8) shows example images of the ORL database.

\subsection{Japanese Female Facial Expression (JAFFE)}

The JAFFE database is composed of 213 images of female facial expression corresponding to 10 distinct subjects. Each image is stored at a resolution of 256X256 pixels and 8-bit gray level. Each subject in the database is represented with 7 categories of expression (angry, disgust, fear, neutral, sadness, happiness and surprise). [21]. Fig (9) shows example images of the JAFFE database.

\subsection{Senthil version 1}

The Senthil database contains 80 colour face images of 5 people (all males), including frontal views (with different angles) of faces with different facial expression. Each person has 16 different images [21]. Fig (10) shows example images of the Senthil version1 database.

\subsection{Face94}

Face94 database consists of 133 individuals, 20 of them are females, and the rest are males. Each individual has 20 face images, with distinct subject containing variation in illumination and facial expression [20]. Fig (11) shows example images of the Face94 database.

\subsection{Yale}

This database consists of 165 greyscale images in bmp format of 15 persons. Each person has 11 face images with different facial expression or configuration: centre-light, with/without glasses, happy, left-light, normal, right-light, sad, sleepy, surprised, and wink [21]. Fig (12) shows example images of the Yale database. 


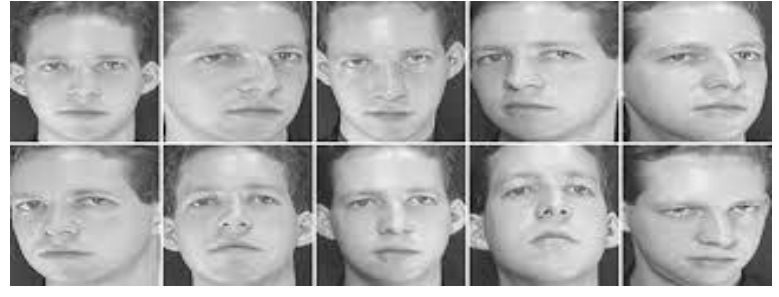

Fig 8: Example images of the ORL database.

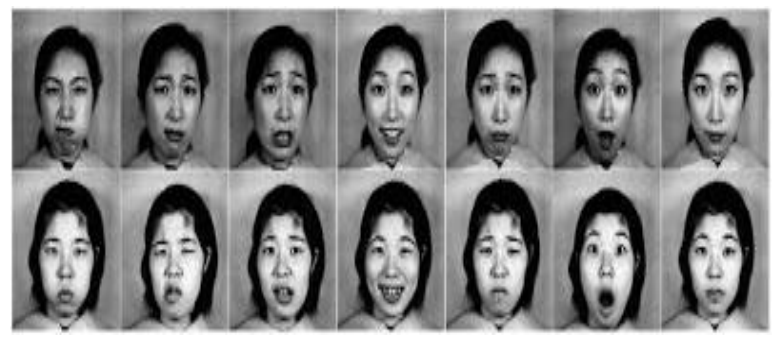

Fig 9: Example images of the JAFFE database.

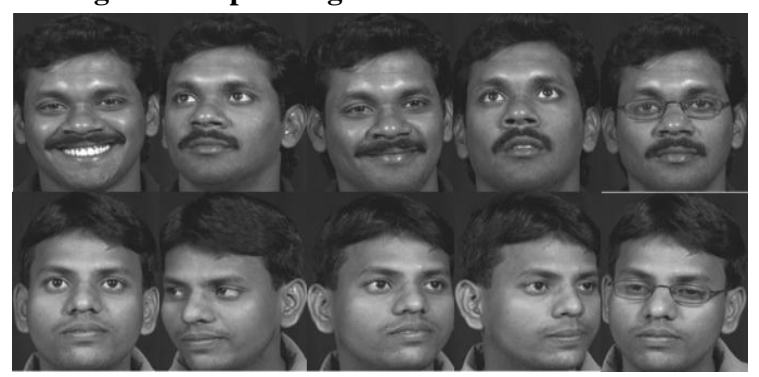

Fig 10: Example images of the Senthil database.
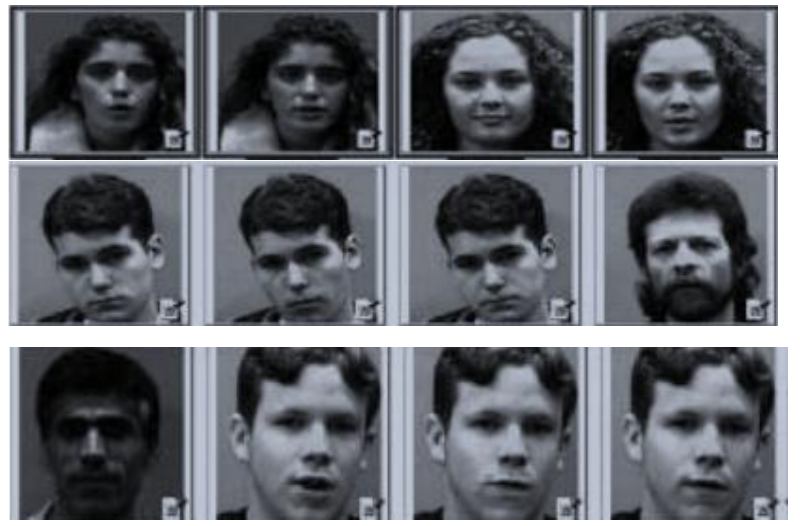

Fig 11: Example images of the Face94 database.
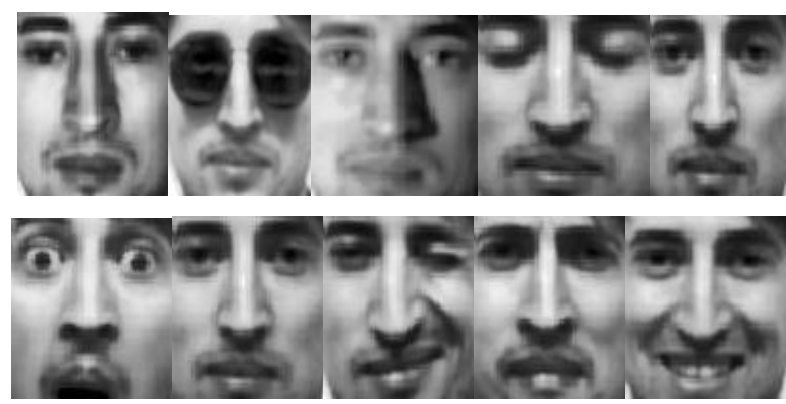

Fig 12: Example images of the Yale database.

\section{ALGORITHM DESCRIPTION}

The objective of the work is to investigate more discriminant features, to enhance the face identification performance. Fig(13) shows the overall process of calculating the CT coefficients, and applying the training, then identifying the test images [20].

\subsection{Pre-processing}

Let Zface and Fface represent the training and testing datasets respectively. First, all images are pre-processed (histogram equalisation) in order to avoid the effect of light source intensity on face images [22].

\subsection{Feature Extraction}

All the face images Zface in training phase are decomposed by utilizing the CT. As a result of performing the CT, coefficients of low and high frequency are obtained in different scales and various directions. The decomposed coefficients are $c 1, c 2-1, c 2-2, \ldots, c l-j$ where $j$ is the number of directions. The coefficients contain most important information of faces that are important in face identification [8]. In this paper five scales of decomposition for CT are used, and then the c1, c2-1 and c2-2 coefficients are considered.

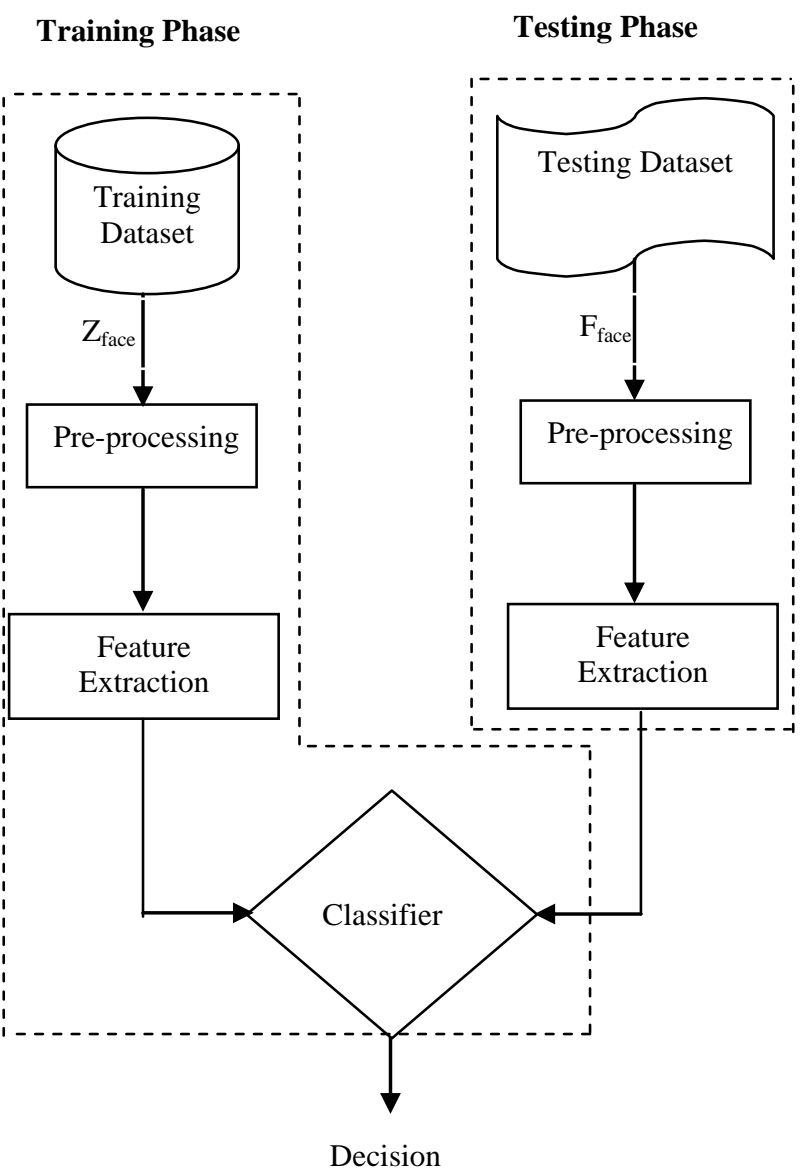

Fig 13: Block diagram of the proposed algorithm.

\subsection{Training Phase}

In the training phase an RGB (Red Green Blue) image is converted into greyscale image, the resulting image is resized to $128 \times 128$ pixels, and then the image is pre-processed using histogram equalisation, in order to avoid the effect of light source intensity on face images [22]. Each image is compressed using the CT to obtain feature matrices with dimensional reduction. In this work $c_{1}, c_{2-1}, c_{2-2}$ coefficients are considered to construct the feature matrix. $c_{1}$ coefficient has $8 \times 8$ (64) pixels. Both $c_{2-1}$ and $c_{2-2}$ have $1 \times 8$ (8) pixels producing a total of 16 pixels. All the coefficients are 
arranged to construct a column vector of $80 \times 1$. The feature image matrix $I=\left[I_{1}, I_{2}, I_{3}, \ldots, I_{P}\right]$ is constructed from the coefficients column vector $I_{i}$. Where $i$ represents the index of the image and the feature matrix represent the input of the classifier in the training phase.

\subsection{Testing Phase}

All testing face images $F_{\text {face }}$ are pre-processed using histogram equalisation then decomposed using the CT. The resultant feature vector is used as input to the classifier, and the index of the output of the highest value corresponds to the index of the identified individual image.

The identification rate of the system can be calculated from the following equations:

$$
p_{k}=\frac{\text { Number of correctly identified test images }}{\text { Total number of test images }}
$$

Where, $p_{k}$ represents the identification rate of the $k^{\text {th }}$ person in the database.

Then, to calculate the overall identification rate of the system an average is computed as:

$$
\text { Average rate }=\left(P_{1}+P_{2}+\ldots+P_{u}\right) / u
$$

Where $u$ is the number of individuals in the database.

\section{RESULTS}

In all experiments the 9-7 biorthogonal filter (referred to as "9-7" filter) is chosen as the pyramidal filter, whilst the ladder filters by Phoong et al. (referred to as "pkva" filter) is used as the directional filter. The two-level sub-bands are used at each level. Five different databases are used to evaluate the algorithm: ORL, JAFFE, Senthil version1, Face24, and Yale. To achieve a direct comparison between the proposed algorithm and other features based methods, the same neural network classifier is used with other types of input feature vectors such as Wavelet and PCA.

To compare the presented algorithm in this paper against Wavelet-based face recognition method and PCA-based face identification method, the experiments are performed on the five databases separately.

The images of each individual person are divided into training images (to train the classifier) and testing images, to calculate the identification rate, each test image is treated independently from other test images of the same person. This means, for example for ORL database there are 200 test images that must be recognised, and the identification rate can be calculated due to Equation (6), then the average of all persons in the database is calculated using Equation (7). For the CT-based method the results are listed in Table.1 that shows the average rate over 10 independent training runs of the neural network classifier, in order to ensure the classifier robustness and to get authenticated results.

To further investigate the proposed algorithm against high variability scenario, the algorithm is evaluated using a composition of two databases. The first one is the JAFFE which has variation in facial expression as shown in Fig (9), and the second is the Senthil which has variation in frontal view as shown in Fig (12). Five subjects from the JAFFE database are chosen, because Senthil database consists of only five subjects. The results indicate that the CT-based classifier also outperforms the wavelet-based method and PCA from training time perspective, due to the high sparsity of the CT.

\section{CONCLUSION}

$\mathrm{CT}$ is a unique transform which can be used to derive the intrinsic features of the face images for identification. CT is not used mainly to compress face image, but is used as a feature extractor. Instead of compression in wavelet and PCA, the CT can provide efficient compression by keeping important information for the identification process. The proposed algorithm is fast and appropriate for real time application, visual surveillance, and robotics systems.

Table. 1 shows that the identification performance from the CT-based classifier outperforms the Wavelet-based classifier and the PCA-based classifier. The reason of the superiority of $\mathrm{CT}$ is its ability to capture directional information, i.e. the CT can effectively represent images of smooth contours. The other benefit of the CT is that it provides a very useful solution when the number of face images in the database is insufficient, because the number of images is increased by a factor of:<smiles></smiles>

Where $L$ represents the number of scales and $l_{j}$ is the order of the DFB at each scale, thus providing more discrimination power to the classification phase. It is clear from Table 1 that the PCA fail, when the same classifier is used, due to the larger feature vector when compared to the CT feature vector. The CT-based method outperforms the Wavelet based classifier as the Contourlet provides efficient compression for full set coefficient and scaled-down version of the image.

Table 1. Identification rate and training time of different feature-based methods using different databases.

\begin{tabular}{|c|c|c|c|c|c|c|}
\hline $\begin{array}{c}\text { Type of } \\
\text { database }\end{array}$ & $\begin{array}{c}\text { Original } \\
\text { image size }\end{array}$ & $\begin{array}{c}\text { No. of } \\
\text { training } \\
\text { images }\end{array}$ & $\begin{array}{c}\text { No. of } \\
\text { Testing } \\
\text { images }\end{array}$ & $\begin{array}{c}\text { Identification Rate } \\
\text { using CT (\%)/ } \\
\text { Training time(sec) }\end{array}$ & $\begin{array}{c}\text { Identification Rate } \\
\text { using Wavelet (\%)/ } \\
\text { Training time(sec) }\end{array}$ & $\begin{array}{c}\text { Identification Rate } \\
\text { using PCA (\%)/ } \\
\text { Training time(sec) }\end{array}$ \\
\hline ORL & $92 \times 112$ & 200 & 200 & $95.8 / 33$ & $92.175 / 47$ & $60 / 51$ \\
\hline JAFFE & $256 x 256$ & 100 & 100 & $100 / 16$ & $89 / 30$ & $90 / 23$ \\
\hline Senthil Version1 & $140 \times 188$ & 40 & 40 & $94.25 / 9$ & $94 / 21$ & $93 / 9$ \\
\hline Faces_24 female & $180 \times 200$ & 200 & 200 & $95 / 30$ & $82.7 / 42$ & $76.1 / 52$ \\
\hline Faces_24 male & $180 \times 200$ & 200 & 200 & $97.5 / 35$ & $76.78 / 34$ & $70.89 / 23$ \\
\hline Yale face & $80 x 80$ & 75 & 90 & $81.1 / 23$ & $92.5 / 25$ & $92.5 / 15$ \\
\hline Senthil\&JAFFE & $256 x 256$ & 40 & 40 & $97.5 / 13$ & \\
\hline
\end{tabular}




\section{REFERENCES}

[1] Yanjun Yan, Rajani Muraleedharan, Xiang Ye and Lisa Ann Osadciw, " Contourlet Based Image Compression for Wireless Communication in Face Recognition System", IEEE International Conference on Communications, May,2008, pp. 505-509.

[2] Walid Riad Boukabou and Ahmed Bouridane " Contourlet-Based Feature Extraction with PCA for Face Recognition" The Institute of Electronics, Communications and Information Technology (ECIT).

[3] Majid Iranpour Mobarakeh, Mehran Emadi, Majid Emadi,"FRBF Neural Network base for Face Recognition using Zernike Moments and PCA", International Journal of Computer Applications, vol. 125, no. 2, pp. 10-14, 2015.

[4] Turk, M., Pentland, A. "Eigenfaces for Recognition," Journal of Cognitive Neuroscience. vol. 3, no. 1, pp. 7186, 1991.

[5] Belhumeur, P.N., Hespanha, J.P., Kriegman, D.J. Eigenfaces vs. Fisherfaces: Recognition using class specific linear projection, IEEE Trans. Pattern Analysis and Machine Intelligence, vol. 19, no. 7, pp. 711720, Jul. 1997.

[6] Bartlett, M.S., Movellan, J.R., Sejnowski, T.J. "Face Recognition by Independent Component Analysis," IEEE Transactions on Neural Netwprks, vol. 13, no. 6, pp. 1450-1464, 2002.

[7] [7] N.G.Chitaliya, A.I.Trivedi, "Feature Extraction using Wavelet-PCA and Neural network for application of Object Classification \& Face Recognition," ICCEA, volume 1, pp.510-514, 2010.

[8] XUEBIN XU, DEYUN ZHANG, XINMAN ZHANG ," An efficient method for human face recognition using nonsubsampled contourlet transform and support vector machine", Optica Applicata, Vol. XXXIX, No. 3, 2009.

[9] Starack J.L.,Candes E.J., Donoho D.L.," The Curvelet transform for image denoising", IEEE Transactions on Image Processing 11(6), 2002, pp. 670-684

[10] Tanaya Mandal, Angshul Majmudar, Q.M.Jonathan W U," Face recognition by Curvelet based feature extraction", International Conference on Intelligent Automation and Robotics, LNCS 4633, 2007, pp. 806817.

[11] Xuebin Xu, Deyun Zhang, Xinman Zhan Zhang,"An efficient method for human face recognition using nonsubsampled Contourlet transform and support vector machine * Optica Applicata, Vol. XXXIX, No. 3, 2009pp 601-615.
[12] DO M.N., Vetterli M., "The Contourlet transform: an efficient directional multiresolution image representation", IEEE Transactions on Image Processing 14(12), 2005, pp. $2091-2106$.

[13] Zhou J., Cunha A.L., M.N. Do.," Nonsubsampled Contourlet transform: construction and application in enhancement", Proceedings - International Conference on Image Processing, ICIP 2005, Vol. 1, pp,469-472

[14] LU Y., Do M.N., "A new Contourlet transform with sharp frequency localization", IEEE International Conference on Image Processing, 2006, pp. 1629- 1632.

[15] Hanglong YU, Shengsheng YU et al., "An image compression scheme based on modified Contourlet transform", Computer Engineering and Application 41(1), 2005, pp. 40- 43.

[16] Ch. Srinivasa Rao, S.Srinivas Kumar, B.N.Chatterji " Content Based Image Retrieval using Contourlet Transform" - ICGST-GVIP Journal, volume 7(3), November 2007.

[17] Jun Yan, Muraleedharan R., Xiang YE, Osadciw L.A., "Contourlet based image compression for wireless communication in face recognition system", IEEE International Conference on Communication, 2008, pp. 505-509.

[18] Mohanad A.M. Abukmeil and Dr. Hatem ELaydi, " Palmprint Recognition System By Using Contourlets Transform And Artificial Neural Network" The Islamic University Of Gaza, 2013

[19] Do,M.N. and Vetterli, M. "The Contourlet Transform: An Efficient Directional Multiresolution Image Representation", IEEE Transactions on Image Processing, vol. 14, no. 12, pp. 2091-2106, 2005.

[20] N.G.Chitaliya and Prof.A.I.Trivedi , "An Efficient Method for Face Feature Extraction and Recognition based on Contourlet Transform and Principal Component Analysis using Neural Network", International Journal of Computer Applications (0975 - 8887) Volume 6- No.4, September 2010.

[21] http://www.face-rec.org/databases/

[22] Ali Ibrahim Abbas, Prof. Dr. Waleed Ameen Mahmoud, Ass. Prof. Nuha Abdul-Saheb Alwan" Face Identification Using Multiwavelet-based Neural Network " A Thesis Submitted to the Department of Electrical Engineering in the University of Baghdad. September 2010. 